CHENG WANG, M.Sc. ${ }^{1}$

E-mail: 20174246020@stu.suda.edu.cn

YIMING WANG, Ph.D. ${ }^{1}$

E-mail: ymwang@suda.edu.cn

CHENG WU, Ph.D. ${ }^{1}$

(Corresponding author)

E-mail: cwu@suda.edu.cn

1 School of Urban Rail Transportation, Soochow University

8 Jixue Road, Suzhou, Jiangsu 215011 - PRC
Information and Communication Technology Original Scientific Paper Submitted: 2 Mar. 2018 Accepted: 24 Oct. 2018

\title{
BAYESIAN SEQUENTIAL LEARNING FOR RAILWAY COGNITIVE RADIO
}

\begin{abstract}
Applying cognitive radio in the railway communication systems is a cutting-edge research area. The rapid motion of the train makes the spectrum access of the railway wireless environment instable. To address the issue, first we formulate the spectrum management of railway cognitive radio as a distributed sequential decision problem. Then, based on the available environmental information, we propose a multi-cognitive-base-station cascade collaboration algorithm by using naive Bayesian learning and agent theory. Finally, our experiment results reveal that the model can improve the performance of spectrum access. This cognitive-base-station multi-agent system scheme comprehensively solves the problem of low efficiency in the dynamic access of the railway cognitive radio. The article is also a typical case of artificial intelligence applied in the field of the smart city.
\end{abstract}

\section{KEY WORDS}

railway; cognitive radio; MAC protocol; naive Bayesian method; spectrum management;

\section{INTRODUCTION}

In the early 1990s, the problem of spectrum scarcity was in its infancy stage, and a large portion of the spectrum was available to be utilized. Wireless networks followed a fixed spectrum assignment policy. As the time goes by, however, the usage of bandwidthhungry wireless applications increases, and the issue of a fixed spectrum assignment policy arises [1]. In order to solve it, the Federal Communications Commission (FCC) approved that unlicensed devices can utilize the licensed spectrum in a way that the licensed user or the primary radio user would not be interfered. After this approval, cognitive radio (CR) appeared, as a promising solution for the fixed spectrum assignment policy by opportunistically utilizing the temporarily unused spectrum, known as spectrum holes or white spaces. The initial idea of $\mathrm{CR}$ is that an unauthorized device (also known as the cognitive user, the secondary user, or the CU) could share a wireless channel with an authorized device (also known as the primary user or the PU) with a dedicated spectrum, and the authorized device would have priority for occupation once it is detected as active [2]. Mitola proposed the concept of cognitive radio in his dissertation [3], and Haykin, with his colleagues, did a lot of research on cognitive radio [4]. We argue that the intelligence of the wireless communication system refers to the ability of learning and forecasting based on artificial intelligence (AI), which could change its working parameters, such as clock frequency, modulation method and transmit power etc., according to external environment. This definition highlights the importance of cognitive radio's external environment learning ability. With the expansion of the CR concept in increasingly dedicated wireless networks, research in this area has focused on application issues, that is, seamless integration of the CR technology and the particularities of dedicated wireless networks [5, 6]. Wireless networks are normally deployed in a distributed manner without a centralized controller. These networks often need to cope with the high volatility of the available spectrum due to network dynamic topology changes, as well as different QoS requirements of the users, such as the railway wireless network.

The structure of wireless communication networks in modern railways is obviously changing from concentrated to distributed. In addition to information exchange between the train and the control center (for example, download the track database, upload the train speed and location), an increasing number of sensor devices have been deployed along with the railway lines and used to collect environmental data, surveillance images and other devices status. A more important development trend is that the railway wireless communication network is expected to provide large-scale broadband data services, which requires the base station to provide a spectrum wide enough to transmit the signal. In fact, the spectrum itself is an expensive and limited resource. [7] In order to cope with mobility, a typical wireless network architecture of the railway 


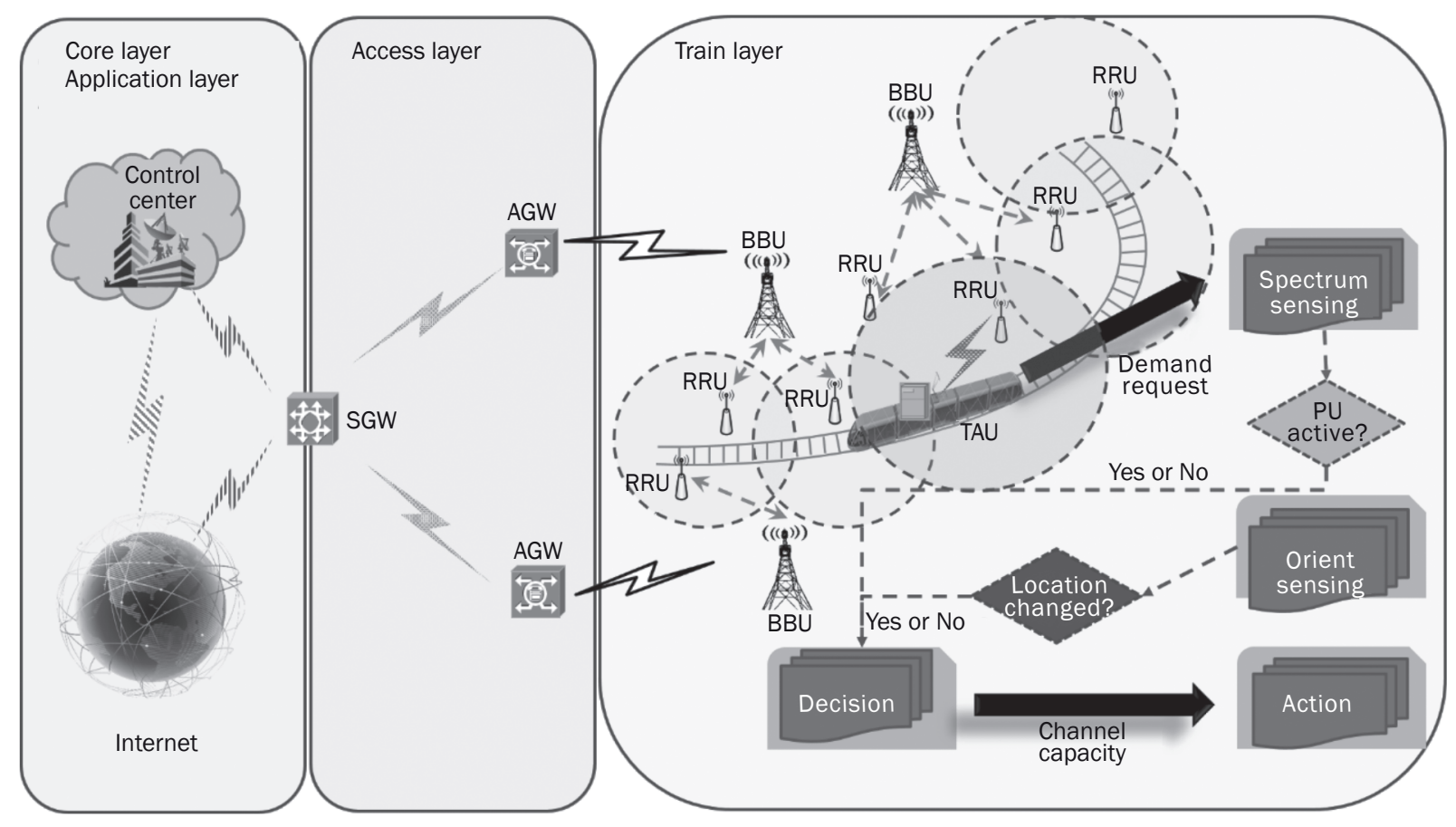

Figure 1 - Railway wireless communication network

$[8,9]$ has been given in Figure 1 . This architecture makes the wireless spectrum environment more complex and unique.

The typical structure of a railway wireless communication network is multi-layered, and composed of four layers: the application layer, the core layer, the access layer and the train layer. The train passenger, the vehicle terminal and the trackside device's sensors use the wireless transmission protocol to communicate with the base station.

- A railway network covers a large geographical area.

The train often encounters different electro magnets and jamming. The channel quality may not be ideal during train transmission due to the inherent unreliability of the wireless channel.

- The train travels along fixed lines and passes quickly through coverages of multiple base stations. The distribution of these base stations is chain-like. Each base station has different authorized devices with their unique spectrum occupancy rules, which makes it difficult to forecast the PU busy-idle station.

- The train which comes into the coverages of some base stations needs to compete with other secondary devices for spectrum occupancy. The CU may suffer a collision with another concurrent unlicensed user such as a wayside.

In this article, we are committed to solve the ubiquitous uncertain problems in railway wireless communication by using the CR technology. Published studies have stuck in the stage of a general idea, and there is no practical radio network model and corresponding experimental verification for cognitive communication
[10]. Our work is motivated by the chain-like deployment of base stations along a railway line across large terrains where the train passes through quickly. Specifically, the train moves along a fixed railway line. The line is usually overlaid by multiple base stations. Bayesian rules are utilized to update the probability distribution of channel switching when additional evidence is observed. Therefore, we mathematically formulate the multi-agent cascade communication problem of these chain-like distributed cognitive base stations using Bayesian learning.

The basic structure of this article is organized as follows. Section 2 outlines the works of decentralized computation and distributed learning in the signal processing community. Section 3 formulates the railway wireless environment and presents the corresponding mathematical model, then further describes the multi-cognitive base station cascade collaborative work under distributed framework. Specific test cases and corresponding experimental results are given in Section 4. This article is summarized in Section 5, and the future research direction is discussed.

\section{RELATED WORK}

\subsection{Railway cognitive radio}

In the existing literature, the wireless communication technologies applied in today's urban railway are: TETRA (trans-European trunked radio), GSM (global system for mobile communications), CDMA (code division multiple access), $4 \mathrm{G}$ (fourth generation mobile communication), Wi-Fi (wireless LAN) and so on. The 
railless wireless communication network is expected to provide large-scale broadband data services, which requires that the base station provides a spectrum wide enough to transmit the signal [11].

In the past decade, the problem of distributed learning has been of high interest within the signal processing community. The latter has been propelled by the technological advances of the wireless sensor networks. The literature primarily includes articles on distributed detection and estimation. In this area, the important issues are information fusion of processed data by sensors and transmission to a fusion center, integration of transceiver design with the design of fusion algorithms, development of methods that are robust to node and link failures, the use of existing machine learning models and algorithms for nonparametric distributed signal processing in wireless sensor networks, consensus building, or, in general, selforganization in networked systems $[12,13]$. The wireless communication technologies in railway can be divided into four modules. The sensing module uses CR spectrum sensing to observe the rail radio environment and obtain the current physical condition using physical sensors (e.g. GPS, acceleration sensors and temperature sensors). Many researchers have contributed to addressing this issue in terms of spectrum sensing and management technique, PU busy-idle station prediction and so on [14-16]. In [17], the Bayesian method in PU prediction is shown. The orientation module studies how to match and meet the specific spectrum strategy and a specific optimization goal, which is considered in $[18,19]$. The decision module provides the required configuration change strategy to accommodate different goals [20]. The action module applies spectrum sharing and mobility to perform a new configuration to change the radio environment, and the multi-agent opportunistic transmission scheduling is modeled [21]. Applications of naive Bayesian learning can be found in diverse fields. Based on these investigations, a cognitive engine (CE) usually does the cognitive work of the rail $\mathrm{CR}$.

The cognitive engine is responsible for learning and predicting the rules of the occupant and the cognitive user's spectrum occupancy in radio operations. In general, the carrier of the cognitive engine is op- tional. Under the railway wireless communication, the base station is usually composed of the baseband unit (BBU) and the wireless remote unit (RRU). The RRUs are usually deployed along the tracks. The BBU connects several RRUs. The BBU and the RRU are used to process the baseband signal and the radio frequency signal, respectively. The communication between the base station and the train passenger is conducted by the RRU, the leak cable and the train access terminal (TAU).

In this process, the base station becomes an indispensable node in the wireless communication network. It has also become a preferred object for the rail cognitive engine. Based on this, we propose a new cognitive base station (CBS) with perceptual and decision-making capabilities. The basic function of the cognitive base station is to discover and to assign the spectrum hole to the cognitive user within the coverage of the cognitive base station while ensuring that the communication of the PU maintains undisturbed.

\subsection{MAC protocols in cognitive radio ad hoc networks}

In $[22,23]$, the MAC protocol in the cognitive radio network generally has the functions of spectrum management, equipment coordination and power control. The design of the MAC protocol faces great challenges because of lack of infrastructure. The existing selforganizing cognitive radio network MAC protocols can be divided into two major categories: negotiated access and non-negotiated access MAC protocols. The negotiated access MAC protocol first uses the specific control channel to achieve the function of the spectrum allocation and then transforms the data. Most of the existing work falls into this category. Some of them use a fixed channel while others use a dynamically changing channel.

Many protocols have been drafted on the cognitive radio network MAC [24-26]. The article proposes a new kind of time structure, which is defined in the MAC protocol. The timing structure is shown in Figure 2. Each beacon interval $(\mathrm{BI})$ is divided into three stages: cognition stage, competition stage and data transmission or channel switching stage.

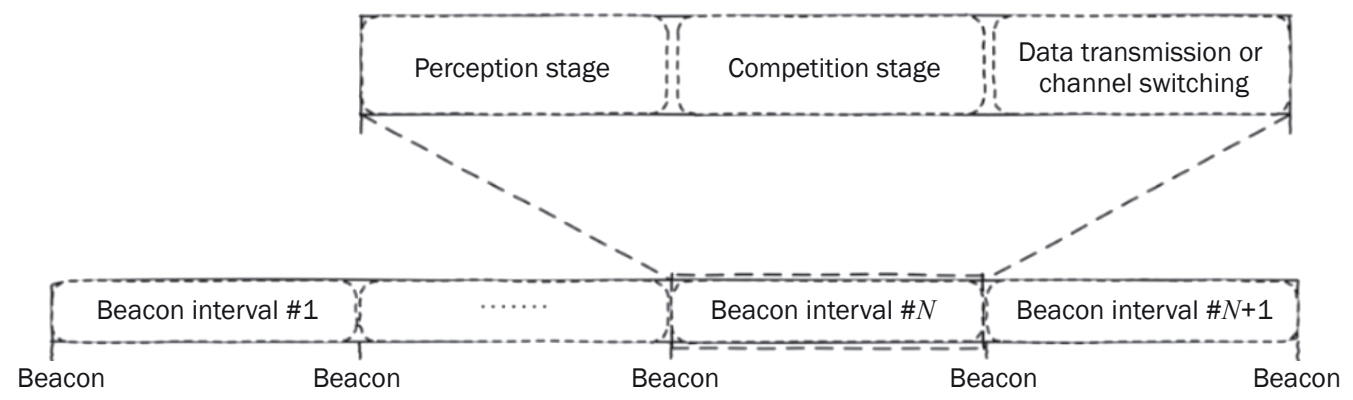

Figure 2 - Timing structure 


\section{METHODOLOGY}

\subsection{System modeling}

A typical cognitive radio network for the railway wireless communication consists of a collection of CBS agents, PUs and train CU agents. The spectrum decision of the CBS agent serves but is also independent of the train CU agents in its coverage area. Each CBS agent has its own PU community and available spectrums. Under normal circumstances, a dedicated frequency band will be allocated to each PU for data transmission.

The channel to which the CBS provides services for the cognitive useris definedas: $c h=\left\{c h_{1}, c h_{2}, \ldots, c h_{i}, \ldots, c h_{N}\right\}$, where $i$ represents the number of channels available on each CBS. The train is scheduled to set out every day, with a specified arrival time, and always with a fixed time along a fixed route. Each beacon interval $(B I)$ experienced by the train as it passes through the CBS is defined as: $B I=\left\{B I_{1}, B I_{2}, \ldots, B I_{j}, \ldots, B I_{M}\right\}$. The elements in the state set $S$ are defined as a combination of all the channel occupancy of the CBS agent and its $B I$, denoted as:

$S=\left\{\begin{array}{cccc}\left(c h_{1}, B I_{1}\right), & \left(c h_{1}, B I_{2}\right), & \ldots & \left(c h_{1}, B I_{M}\right) \\ \left(c h_{2}, B I_{1}\right), & \left(c h_{2}, B I_{2}\right), & \ldots & \left(c_{1}, B I_{M}\right), \\ \vdots & \vdots & & \vdots \\ \left(c h_{i}, B I_{1}\right), & \left(c h_{i}, B I_{2}\right), & \ldots & \left(\operatorname{ch}_{i}, B I_{M}\right), \\ \vdots & \vdots & & \vdots \\ \left(c h_{N}, B I_{1}\right), & \left(c h_{N}, B I_{2}\right) & \ldots & \left(c_{N}, B I_{M}\right)\end{array}\right\}$

The action set $C$ contains $N x M$ kinds of actions, allowing the $\mathrm{CU}$ to transmit or allocate the $\mathrm{CU}$ to the other channels, as shown in Formula 2.

$C=\left\{\begin{array}{ccccc}c_{11}, c_{12}, & \ldots, & c_{1 j}, & \ldots, & c_{1 M} \\ c_{21}, c_{22}, & \ldots, & c_{2 j}, & \ldots, & c_{2 M} \\ \vdots & & & & \vdots \\ c_{i 1}, c_{i 2}, & \ldots, & c_{i j} & \ldots, & c_{i M} \\ \vdots & & & & \vdots \\ c_{N 1}, c_{N 2} & \ldots, & c_{N j}, & \ldots, & c_{N M}\end{array}\right\}$

s.t. $c_{i j}=\left\{\begin{array}{l}1, \text { transmit data or access in } \\ 0, \text { else }\end{array}\right.$

$0 \leq \sum_{i=1}^{N} c_{i j} \leq 1,(0 \leq i \leq N, 0 \leq j \leq M)$

This is because the action behaviors of these CBS agents do not occur simultaneously, and because they occur in cascade. The utility function is defined to satisfy the goals of minimizing the blocking rate and minimizing the channel switching over all CBSs in the cognitive base-station multi-agent system.

The two-state Markov chain model is widely used in the literature to model the PR user activity. The two states in this model are the BUSY state and the IDLE state. The BUSY state indicates that the channel is currently occupied by the PU and is unavailable for the $\mathrm{CU}$. The IDLE state indicates that the channel is free and there is no PU activity on the channel. The PU changes from the current state to the next state according to the current state and the transition probability, while it has nothing to do with the previous state, that is, the system is not post-effect. If the channel is currently in the BUSY state, the probability that the next state of the channel will also be BUSY is $p$, while the probability of the next state to be IDLE is 1- $p$. If the channel's current state is IDLE, then its probability of remaining IDLE again is 1- $q$ while the probability that in the next state, it will be in the BUSY state and occupied by the PU user is $q$. In this model, we take a uniform distribution of this transition probability [0.0-1.0 random].

$T_{\text {busy }}^{k}$ represents the exponential distribution time after the state transitions to the busy state, $T_{i d l e}^{k}$ represents the exponential distribution time after the channel transitions to the idle state, and the exponential rate parameters are $\lambda_{\text {busy }}$ and $\lambda_{\text {idle }}$, that is, the occupied state or the idle state. The occupancy state duration function is $f\left(T_{b u s y}^{k} ; \lambda_{b u s y}\right)$, and the idle state duration function is $f\left(T_{i d l e}^{k} ; \lambda_{\text {idle }}\right)$.

$\left\{\begin{array}{l}f\left(T_{\text {busy }}^{k} ; \lambda_{\text {busy }}\right)=\lambda_{\text {busy }} e^{-\lambda_{\text {busy }} T_{\text {busy }}^{k}} \\ f\left(T_{\text {idle }}^{k} ; \lambda_{\text {idle }}\right)=\lambda_{\text {idle }} e^{-\lambda_{\text {idle }} T_{\text {idle }}^{k}}\end{array}\right.$

\subsection{Distributed sequential decision using naive Bayesian learning}

Now we use the multi-agent system with naive Bayesian learning ability to solve the distributed sequential decision problem for spectrum management of multiple CBSs. For the given railway wireless communication network, we set that the goal of the CBS spectrum management is to minimize the blocking rate of the entire network and the number of channels switching to meet the needs of rapid train movement.

In order to deal with the problem of spectrum allocation in a single CBS by using naive Bayesian learning, we define a sample feature $X$, where $X$ is a feature vector, and the dimension of $X$ is set to $M$. According to the total probability formula, the features are independent of each other, so the formula can be shown as follows:

$P\left(y=\tilde{c}_{k} \mid x\right)=\frac{\prod_{i=1}^{M} p\left(x^{i} \mid y=\tilde{c}_{k}\right) p\left(y=\tilde{c}_{k}\right)}{\sum_{k} p\left(y=\tilde{c}_{k}\right) \prod_{i=1}^{M} p\left(x^{i} \mid y=\tilde{c}_{k}\right)}$

In the article, the naive Bayesian model is a repetitive training process. The input is represented by the vector $x_{i}^{(N)}$, where $i$ represents the $i$-th feature, and the superscript represents the dimension of the feature:

$X_{i}^{(N)}=\left(x_{i}^{(1)}, x_{i}^{(2)}, \ldots, x_{i}^{(N)}\right)$

Because the train is running at high speed in a linear environment, due to various geographical environments, the set of channels available for service is 


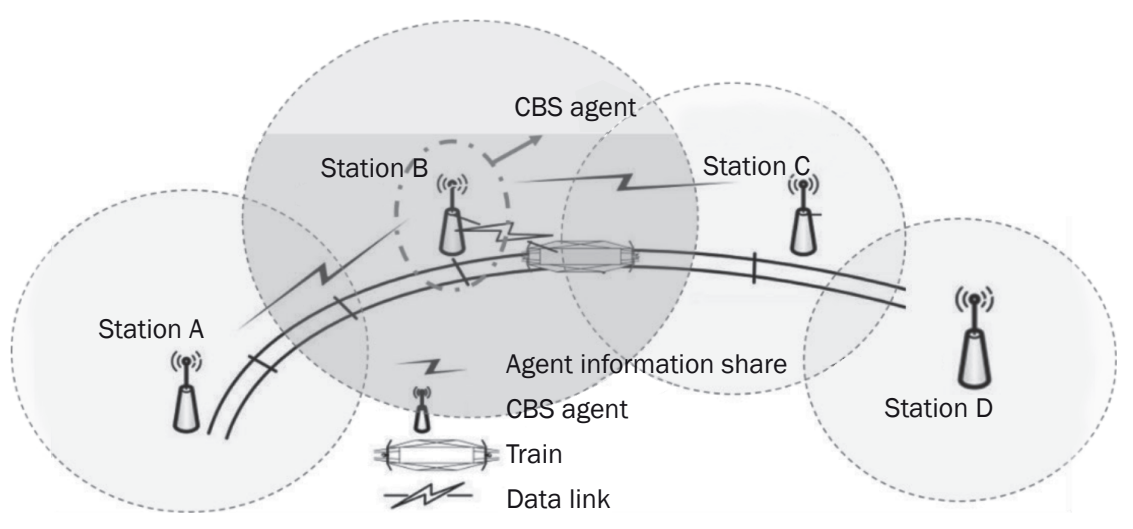

Figure 3 - Multi-cognitive base station

different among CBSs. Obviously, when a cognitive user chooses a suitable service channel, the CUs should not only avoid interfering with the quality of service of the primary user, but also the interference between two adjacent CBSs. We assume that there are several adjacent CBSs as shown in Figure 3. It can be seen that the $C B S_{B}$ and the $C B S_{A}, C B S_{C}$ have overlapping coverage areas, thus they cannot share a channel. The $C B S_{A}$ and $C B S_{D}$ do not overlap, so they can share a channel. In a multi-CBS system, each CBS broadcasts its personal information to the neighboring CBS, i.e., channel occupancy and beacon interval messages. Here we set these two features (channel and $B I)$ as interdependent inputs. So, the number of input parameter is $N \mathrm{x} M$.

There are $N \times M$ kinds of output categories. In order to distinguish this category with a feature, the output in a certain $B I$ would be expressed as:

$Y=\left\{\tilde{c}_{1}, \tilde{c}_{2}, \tilde{c}_{3}, \ldots, \tilde{c}_{i}, \ldots, \tilde{c}_{N}\right\}$

Next, we would calculate the conditional probability of the characteristic $x_{i}^{(k)}$ in the case of different categories. The prior probabilities of the category $y$ can be calculated from the training set. Through the statistics of the training set, we can draw the conditional probability of the corresponding feature on each category. A large number of training sets $\left\{\left(x_{i}^{(1)}, y_{1}\right),\left(x_{i}^{(2)}, y_{2}\right), \ldots,\left(x_{i}^{(N)}, y_{N}\right)\right\}$ will be generated during the process of the train passing through the CBS. For a given sample, we first need to calculate the likelihood estimate of the prior probability $p\left(y=\tilde{c}_{k}\right)$ in Equation 7:

$p\left(y=\tilde{c}_{k}\right)=\frac{\sum_{i}^{N} I\left(y_{N}=\tilde{c}_{k}\right)}{N}$

$I(\cdot)$ is an instruction function; thus, if the feature or category occurs, $I(\cdot)=1$, otherwise the instruction function is 0 .
Then we have to calculate the conditional probability in the molecule. In view of a single base station, the number of dimensions is $N \times M$. In a certain feature $S_{i j}=\left(c h_{i}, B I_{j}\right)$, where $x_{i}^{j}$ is expressed as $a_{i j}$, the conditional probability under the given category $\tilde{c}_{k}$ is:

$P\left(x^{i}=a_{i j} \mid y=\tilde{c}_{k}\right)=\frac{\sum_{i=1}^{N} I\left(x_{i}^{j}=a_{i j}, y_{N}=\tilde{c}_{k}\right)}{\sum_{i=1}^{N} I\left(y_{N}=\tilde{c}_{k}\right)}$

A given unclassified new instance $X$ can be calculated by the probability above. The posterior probability $P\left(y=\tilde{c}_{k} \mid X\right)$ of the instance would be obtained. Because in all the categories the denominator values in Equation 4 are the same, we just need to calculate the fractional molecules. The specific steps go as follows.

First, calculate the probability that the category equals $\tilde{c}_{k}$, as shown in Formula 9.

$P\left(y=\tilde{c}_{k} \mid X\right)=p\left(y=\tilde{c}_{k}\right) \prod_{j=1}^{n} P\left(X^{(j)}=x^{(j)} \mid y=\tilde{c}_{k}\right)$

Then, determine what kind of category should be selected by:

$y=\arg \max _{\bar{c}_{k}} p\left(y=\tilde{c}_{k} \mid X\right)$

Therefore, we get the result of the classification which means the CU's action. However, a small problem that remains unsolved is how to obtain the prior probability if the sample is zero. In Equations 7 and 8, in order to avoid the denominator being zero, we add a constant $\lambda$ to both the numerator and the denominator, where $\lambda=1$. This is Laplace smooth. The updated equations are as follows:

$$
\begin{gathered}
p\left(y=\tilde{c}_{k}\right)=\frac{\sum_{i=1}^{N} I\left(y_{N}=\tilde{c}_{k}\right)+\lambda}{N+K \lambda} \\
P\left(x^{j}=a_{i j} \mid y=\tilde{c}_{k}\right)=\frac{\sum_{i=1}^{N} I\left(x_{i}^{j}=a_{i j}, y_{N}=\tilde{c}_{k}\right)+\lambda}{\sum_{i=1}^{N} I\left(y_{N}=\tilde{c}_{k}\right)+L_{j} \lambda}
\end{gathered}
$$




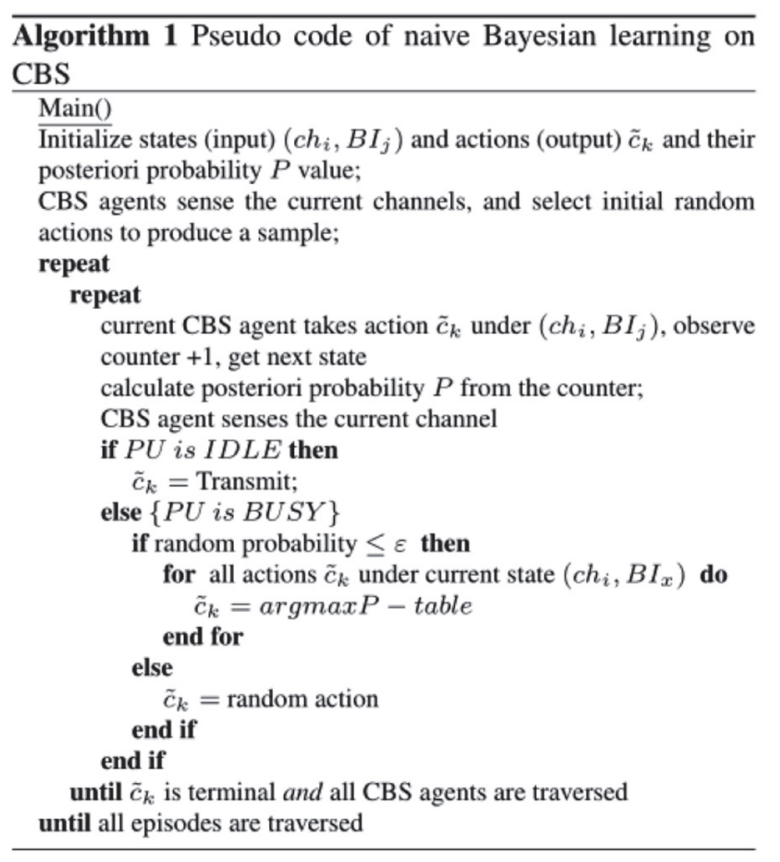

Figure 4 - Algorithm 1: Pseudo code of naive Bayesian learning on $C B S$

Where $K$ is the number of categories, and $L_{j}$ is the maximum value of the feature of the $j$-th dimension. The specific naive Bayesian learning algorithm implementation of multiple CBS agents is shown as Algorithm 1 (Figure 4).

\section{EXPERIMENTAL EVALUATION}

The construction of the railway environment, the functionality of the cognitive radio, learning and the decision mechanisms of agents are realized by an independent $\mathrm{C}++$ module, in which the protocols of the physical layer, the link layer and the network layer are modified accordingly. The agent system module provides the architecture of the multi-agent, which consists of agent declarations, agent cooperation mechanisms and agent communication protocols. The

Table 1 - Simulation parameters learning module describes several typical learning algorithms and some common functions, for the calling from the agent system module.

The PU-activity module uses the two-state Markov model to define the activities of the PU, such as transmission distance, interference range, spectrum occupation rules and PU locations which are shown in Figure 5a. The gray part represents the train running over the coverage of the previous CBS, or not yet reaching the next, or the channel being occupied by the nearby base stations. The red and blue portions represent the occupation of the PU.

The railway wireless communication network environment repository contains information on transmission power settings, train speed and location, base station locations and coverages, and different network protocols for railway environments. More details can be found in our previous publication.

Based on the above simulation platform, we assume that there are 10 channels in the system. Each CBS covers three or four channels. The parameters of the train's operation and the cognitive radio system are shown in Table 1, where the base station coverage area is converted to a linear distribution. All experiments are conducted on average values to reduce contingency.

In order to evaluate the proposed scheme, we use the railway wireless communication network simulation platform and compare the performance of the CBS multi-agent scheme based on naive Bayesian learning (CBS-BL) with the base station scheme using the round robin mechanism (BS-RR). The BS-RR scheme adopts the following principle: once the PU occupies the spectrum, the agent switches to the next channel. This method is simple, easy to implement and will not "starve to death". $\varepsilon$ is a greedy parameter, representing the probability of using Bayesian learning. When the random number is larger than $\varepsilon$, the system uses a random mechanism, otherwise, it will learn by

\begin{tabular}{||l|c||}
\hline \multicolumn{1}{|c|}{ Number of CBSs } & 3 \\
\hline \hline CBS coverage (X: initial position; Y: exit position) & X:50 m-230 m $\backslash \mathrm{B}: 200 \mathrm{~m}-376 \mathrm{~m} \backslash \mathrm{C}: 356 \mathrm{~m}-471 \mathrm{~m}\}$ \\
\hline The average speed of train operation & $301.76 \mathrm{~km} / \mathrm{h}$ \\
\hline Number of CBS channels & 10 \\
\hline Number of primary users & 10 \\
\hline Number of cognitive users & 1 \\
\hline Beacon interval & $1 \mathrm{~ms}$ \\
\hline The location where cognitive user requests data transmission & $200 \mathrm{~m}$ [base station B] \\
\hline Cognitive user transmission time & $2100 \mathrm{~ms}$ \\
\hline
\end{tabular}


naive Bayesian learning. We consider using two performance indicators, such as blocking rate and the number of spectra switching for the results of verification.

Figure 5a shows the occupancy of the primary user from the train's starting position to 2100 beacon intervals. The gray part represents the train running over the coverage of the previous CBS, or it not yet reaching the next CBS, or the channel being occupied by the nearby base stations. The ordinary portion represents the information of the CBS's own channel and beacon interval, or the received neighboring base stations.

Figure $5 b$ shows the average probability of the blocking rate using our test scenarios in the simulation platform. Each epoch consists of 2100 Bls. During this period, the system calculates the prior probability. In the graph, the red curve represents the performance of the CBS-BL scheme, which decreases with the increase of epochs, but finally becomes stable. The blue curve represents the BS-RR. After approximately 1000 epochs, the CBS-BL scheme tended to reach $17 \%$ of the average probability of the blocking rate, while the BS$\mathrm{RR}$ remained at approximately $28 \%$.

Figure $5 c$ shows the average number of spectrum handovers using our test scenarios in the simulation platform. The red curve represents the performance of the CBS-BL scheme, which maintains good ability with the increase of epochs and finally becomes stable. The BS-RR switches its channel at each beacon interval (the number of switching channels is 2100). The results show that naive Bayesian learning can help the CBS multi-agent system to mine the spectrum hole in the railway wireless communication environment effectively, avoid the wrong actions of channel switching, and improve the efficiency of the spectrum management.

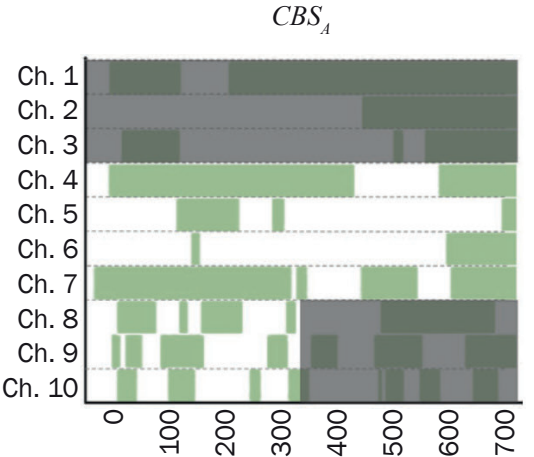

$B I$
$C B S_{B}$

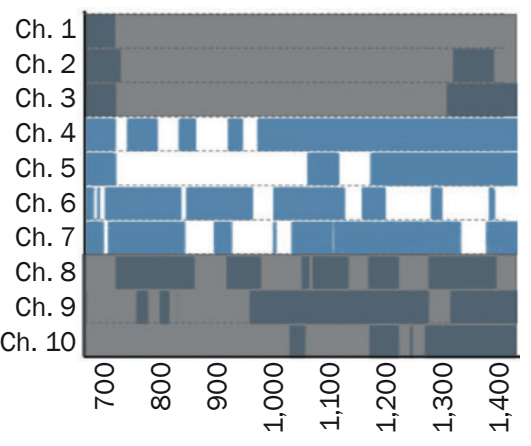

$B I$

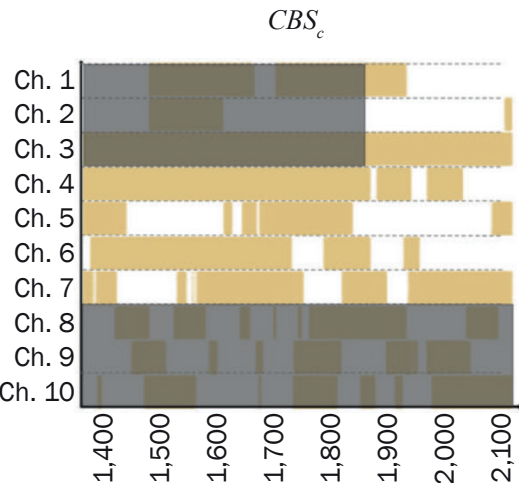

$B I$

Spectrum in use Ch. = Channel

a) Primary user model

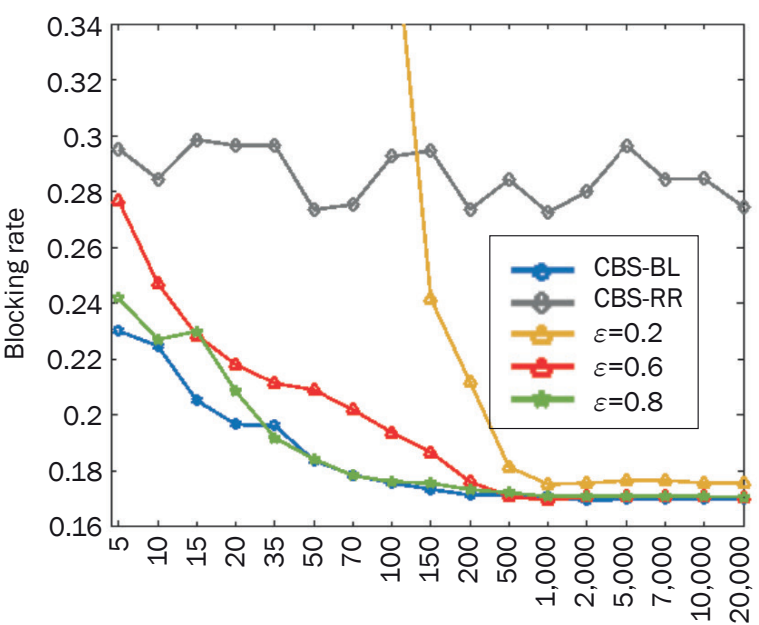

The number of epoch

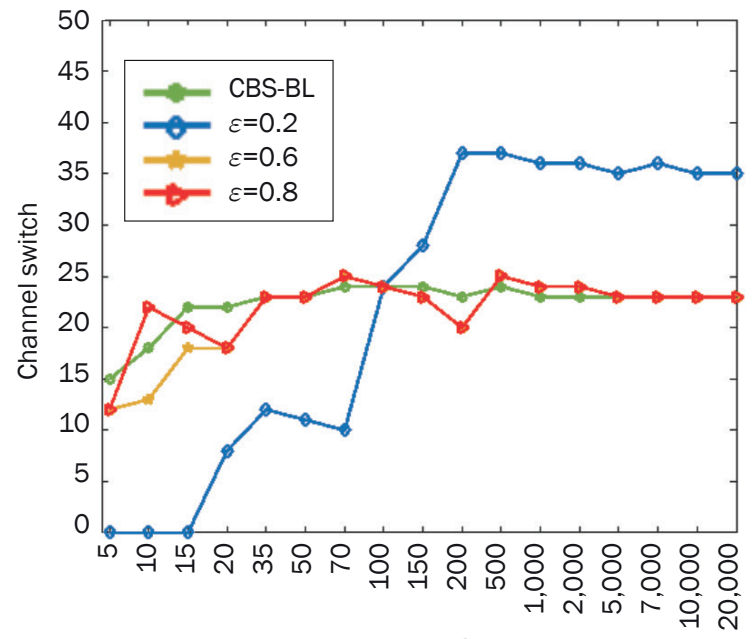

The number of epoch

b) Blocking rate

c) Channel switching times

Figure 5 - Experimental evaluation 


\section{CONCLUSION}

Railway cognitive radio is the most recently launched cutting-edge research and is still in its infancy stage. In this article, we propose a multi-agent railway communication modeling for channel accessibility, using a fusion of prior and validation information. It is a typical case of artificial intelligence applied in the field of transportation to deal with the increasingly complex behaviors of railway wireless communication in order to meet communication requirements and improve communication efficiency. We focus on addressing two major railway wireless communications issues, that is, the railway radio spectrum opportunistic access and the spectrum efficiency improvement in fast movement. Experimental results show that the cognitive base-station multi-agent system significantly improves the data transmission performance and greatly reduces the number of wireless spectra switching.

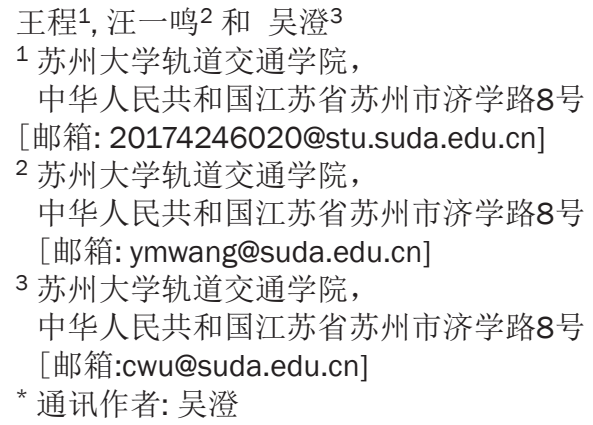

\section{摘要}

在铁路通信系统中应用认知无线电是一个新兴研究领 域。列车的快速运动使得铁路无线环境的频谱接入具有不 稳定性。为了解决这个难点, 首先, 我们将铁路认知无线 电的频谱管理表示为为分布式顺序决策问题。紧接着, 基 于可用的列车环境信息, 我们提出了一种基于朴素贝叶斯 学习和智能体理论的多认知基站级联协作算法。最后, 我 们通过仿真实验结果表明该模型对提高频谱接入性能的可 用性。该认知基站多智能体系统方案全面解决了铁路认知 无线电动态接入效率低的问题。本文也是应用于智慧城市 领域的典型人工智能案例。

\section{关键词}

铁路, 认知无线电, MAC协议, 朴素贝叶斯方法, 频谱管 理

\section{REFERENCES}

[1] Saleem Y, Rehmani MH. Primary radio user activity models for cognitive radio networks: A survey. Journal of Network \& Computer Applications. 2014;43(1): 1-16.

[2] Akan ÖB, Karli OB, Ergul O. Cognitive Radio Sensor Networks. Network IEEE. 2009;23(4): 34-40.

[3] Mitola J, Maguire GQ, Jr. Cognitive radio: making software radios more personal. IEEE Pers Commun. 1999;6(4): 13-8.

[4] Haykin S. Cognitive radio: brain-empowered wireless communications. IEEE Journal on Selected Areas in Communications. 2005;23(2): 201-20.

[5] Wang J, Ghosh M, Challapali K. Emerging Cognitive Radio Applications: A Survey. Communications Magazine IEEE. 2011;49(3): 74-81.

[6] Sun D, Song T, Gu B, Li X, Hu J, Liu M. Spectrum Sensing and the Utilization of Spectrum Opportunity Tradeoff in Cognitive Radio Network. IEEE Communications Letters. 2016;20(12): 2442-5.

[7] Fokum DT, Member S, Frost VS. A Survey on Methods for Broadband Internet Access on Trains. IEEE Communications Surveys \& Tutorials. 2010;12(2): 171-85.

[8] Yang J, Zhao H. Enhanced Throughput of Cognitive Radio Networks by Imperfect Spectrum Prediction. IEEE Communications Letters. 2015;19(10): 1738-41.

[9] Yin H, Han B, Li D, Lu F. Modeling and Application of Urban Rail Transit Network for Path Finding Problem. 2011;124: 689-95.

[10] Wu C, Wang Y. Cognitive Communication in Rail Transit: Awareness, Adaption, and Reasoning. It Professional. 2017;19(4): 45-54.

[11] Hodge VJ, O'Keefe S, Weeks M, Moulds A. Wireless Sensor Networks for Condition Monitoring in the Railway Industry: A Survey. IEEE Transactions on Intelligent Transportation Systems. 2015;16(3): 1088106.

[12] Braca P, Marano S, Matta V, Willett P. Asymptotic optimality of running consensus in testing binary hypotheses. IEEE Transactions on Signal Processing. 2010;58(2): 814-25.

[13] Dimakis AG, Kar S, Moura JMF, Rabbat MG, Scaglione A. Gossip Algorithms for Distributed Signal Processing. Proceedings of the IEEE. 2010;98(11): 1847-64.

[14] Kim I, Kim D. Distributed spectrum sensing and access with secondary channel quality in cognitive radio networks. 2010;1: 541-6.

[15] Hossain E, Niyato D, Han Z. Dynamics of Multiple-Seller and Multiple-Buyer Spectrum Trading in Cognitive Radio Networks: A Game-Theoretic Modeling Approach. IEEE Transactions on Mobile Computing. 2009;8(8): 1009-22.

[16] Canberk B, Akyildiz IF, Oktug S. Primary User Activity Modeling Using First-Difference Filter Clustering and Correlation in Cognitive Radio Networks. IEEE/ACM Transactions on Networking. 2011;19(1): 170-83.

[17] Liu X, Chong EKP, Shroff NB. Opportunistic transmission scheduling with resource-sharing constraints in wireless networks. Selected Areas in Communications IEEE Journal on. 2001;19(10): 2053-64.

[18] Choi S, Park H, Hwang T. Optimal Beamforming and Power Allocation for Sensing-Based Spectrum Sharing in Cognitive Radio Networks. IEEE Transactions on Vehicular Technology. 2014;63(1): 412-7.

[19] Li HJ, Qi Z, editors. Tradeoff between utilization and collision in cognitive radio. International Conference on Wireless Communications \& Signal Processing; 2009.

[20] Siris VA. Spectrum Assignment in Cognitive Radio Networks: A Comprehensive Survey. IEEE Communications Surveys \& Tutorials. 2013;15(3): 1108-35. 
[21] Liu X, Chong EKP, Shroff NB. Opportunistic transmission scheduling with resource-sharing constraints in wireless networks. Selected Areas in Communications IEEE Journal on. 2001;19(10): 2053-64.

[22] Peh ECY, Liang YC, Guan YL, Zeng Y, editors. Optimization for Cooperative Sensing in Cognitive Radio Networks. IEEE Wireless Communications and Networking Conference; 2007.

[23] YucekT,ArslanH.Asurveyofspectrumsensingalgorithms for cognitive radio applications. IEEE Communications
Surveys \& Tutorials. 2009;11(1): 116-30.

[24] Akyildiz IF, Lee WY, Chowdhury KR. CRAHNs: Cognitive radio ad hoc networks. Ad Hoc Networks. 2009;7(5): 810-36.

[25] Cormio C, Chowdhury KR. A survey on MAC protocols for cognitive radio networks. Ad Hoc Networks. 2009;7(7): 1315-29.

[26] Domenico AD, Strinati EC, Benedetto MGD. A Survey on MAC Strategies for Cognitive Radio Networks. IEEE Communications Surveys \& Tutorials. 2012;14(1): 21-44. 